\title{
Protée
}

\section{Les lieux de la mélancolie chez Lani Maestro}

\section{Marie Fraser}

Volume 28, numéro 3, 2000

Mélancolie entre les arts

URI : https://id.erudit.org/iderudit/030602ar

DOI : https://doi.org/10.7202/030602ar

Aller au sommaire du numéro

Éditeur(s)

Département des arts et lettres - Université du Québec à Chicoutimi

ISSN

0300-3523 (imprimé)

1708-2307 (numérique)

Découvrir la revue

Citer cet article

Fraser, M. (2000). Les lieux de la mélancolie chez Lani Maestro. Protée, 28(3), 30-42. https://doi.org/10.7202/030602ar d'utilisation que vous pouvez consulter en ligne.

https://apropos.erudit.org/fr/usagers/politique-dutilisation/ 


\section{Lani \\ Maestro}

Lani Maestro est née à Manila aux Philippines. Elle a émigré au Canada en 1982. Son travail a été présenté au Québec, au Canada ainsi qu'à l'étranger. Elle est cofondatrice de Harbour Magazine of Art and Everyday Life (1991-1994), une revue consacrée aux œuvres et aux écrits d'artistes, et coéditrice des Editions Burning depuis 1992. Parmi ses expositions individuelles, on retrouve To dream sleep, présentée au Centre for New Media et au Centre culturel canadien à Paris (1998), Dream of the other, à la Centrale à Montréal, The Passion of ear, au Centre d'art contemporain de Basse-Normandie en France (1997) et Cradle a Art in General à New York (1996). Lani Maestro a également participé à la $11^{\text {th }}$ International Biennale de Sydney en Australie (1998), à la $5^{\text {th }}$ Biennale d'Istambul et à l'exposition Traversées/Crossings au Musée des beauxarts du Canada (1997). 


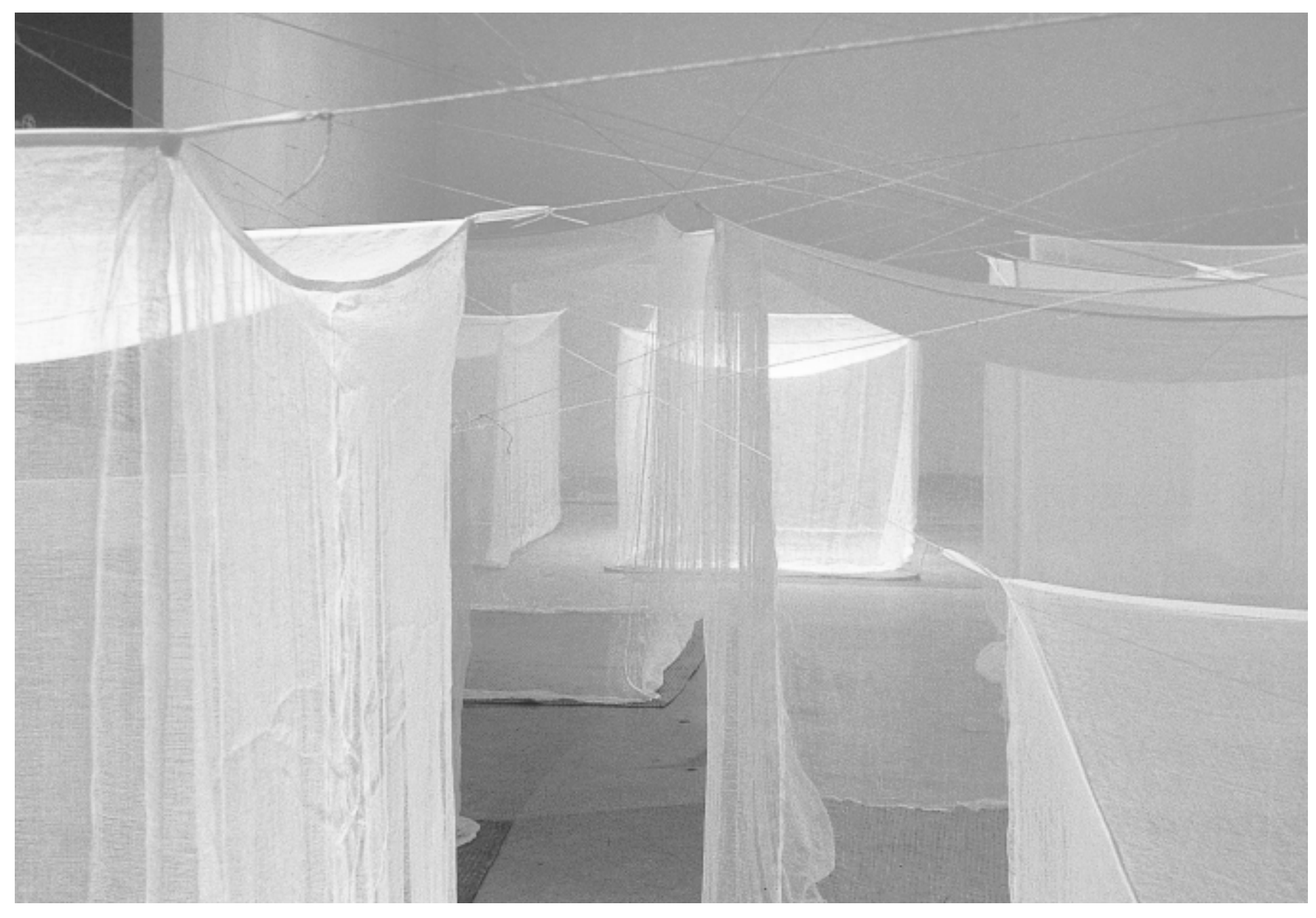




\title{
les lieux de la mélancolie chez Lani Maestro
}

\author{
PAR MARIE FRASER
}

Toujours resterais-je séduit par la grâce avec laquelle l'art de Lani Maestro m'entraîne dans un lieu de mélancolie et de compassion, une expérience émotionnelle inscrite dans les menus détails de ses profondeurs soigneusement ouvragées. Art qui prend racine dans le contrôle de sa destinée et dans le dialogue, l'œuvre de Lani Maestro nous mène à des explorations délicates des thèmes très intimes que sont le chez-soi, le lieu et la mémoire. ${ }^{1}$

Les installations de Lani M aestro nous «entraînent» dans un espace de la mélancolie provoquée par la perte des certitudes qui est au fond des réflexions contemporaines sur les pratiques culturelles et l'identité. L'intermédialité joue, chez elle, autant entre les médiums et les médias qu'entre les corps (nous qui regardons et faisons l'expérience de l'œuvre) et fait fuir toute certitude, abolissant toute possibilité d'unité et d'identification, toute chose donnée d'avance et tout a priori. Elle aurait pour effet de nous dérouter et, parce qu'elle a cette potentialité de fuir, elle nous conduirait à une lecture politique et à des réflexions sur l'identité, l'appartenance, la mémoire. Les œuvres qui sont présentées et commentées ici mettent en évidence cette capacité du travail de Lani $M$ aestro à nous déstabiliser, à nous dérouter de manière à nous inciter à remettre en question nos certitudes. Elles sont le lieu d'une séparation et invitent à la reconnaissance d'une perte et d'une blessure identitaire, un renoncement à soi et à la stabilité du voir. La mélancolie viendrait se loger dans cette fuite provoquée par I'intermédialité. C'est cette particularité à déstabiliser notre perception et notre appréhension de l'œuvre que je voudrais examiner en regard de ces deux notions.

Ces lieux de la mélancolie qui résultent de l'intermédialité ont peut-être moins d'affinité avec la pensée freudienne qu'avec celle de Walter Benjamin. Certes, dans son célèbre article Deuil et mélancolie écrit en 1917, Freud pose les limites de la relation d'objet et l'importance du renoncement comme caractérisant ses deux états. La mélancolie, tout comme le deuil, serait causée par une «intolérance à la perte de l'objet». C'est plutôt du côté de Walter Benjamin que I'on retrouve une pensée de la mélancolie proche de ce qui est en jeu dans le contexte intermédiatique et dans le travail de Lani Maestro. La mélancolie, en plus de se voir attribuer des fonctions presque heuristiques, se fait subrepticement politique. O n sait le rôle fort important qu'elle joue: de la réhabilitation de l'allégorie, qui trouve sa source dans la tristesse, à l'ambiguïté de la tradition dans la modernité et, enfin, à une philosophie de l'histoire qui renonce à tout positivisme et réfute le progrès et la linéarité. L'expérience benjaminienne est bien la prise en charge d'un double deuil : deuil de l'Histoire et de la modernité, tel que le souligne Catherine Perret ${ }^{2}$. La «blessure» causée par cette perte ou par cette séparation y prend toutefois une dimension philosophique et politique. À travers le mouvement dialectique qu'il voit comme inhérent à I'histoire de la mélancolie depuis Aristote, telle que décrite dans L'O rigine du drame baroque allemand, Walter Benjamin se trouve à affirmer cette nécessité du politique ${ }^{3}$. À la mélancolie, il oppose en effet la résistance et le détachement plutôt que le repli sur soi et désarticule l'attitude de passivité et de démission provoquée par le sentiment de perte qui caractérise le mélancolique. 
Lani Maestro rappelle cette mélancolie benjaminienne, ses œuvres articulent autant la perte que sa prise en charge et sa guérison. Elle «exprime la perte, l'éloignement et la disparition, mais aussi le souvenir et la guérison », comme l'a déjà montré Germain Koh: «la prise de conscience de l'identité coïncide avec sa disparition ${ }^{4}$. Ses installations sont issues des rapports hybrides mettant en relation différents matériaux, souvent éphémères, des références textuelles, différents médiums et médias technologiques (la photographie, l'image vidéo et le son par exemple), mais Lani M aestro donne à ce mouvement entre les médiums une dimension précaire et incertaine nous obligeant à repenser notre rapport à l'œuvre. Elle arrive à exprimer cette fragilité et cette précarité en nous mettant en présence de lieux et de formes extrêmement dépouillés et minimales, comme avec I'installation Le Souffle blessé (A Wound in the lung, 1994). N on sans rappeler Earth Work de Walter de Maria, cette chambre de terre est comme «un trou, un vide, [qui] devient un lieu d'appartenance, telle une matrice ou encore une tombe ${ }^{5}$. II s'agit d'un lieu vide et plein tout à la fois: la pièce est vide, il n'y a que de la terre, en même temps qu'elle en est pleine, elle est impénétrable, mais tout aussi pénétrante. La terre vient ici exprimer l'idée d'origine et d'appartenance, la terre-mère et le souffle, en même temps que la chambre fermée nous place à une certaine distance. Contraints de regarder cette chambre depuis l'embrasure d'une porte, nous sommes confrontés à cette séparation que le titre donne comme une blessure. La terre remplit le lieu jusqu'à mijambe et l'assourdit, le rendant encore perméable à toute présence. L'espace est lisse, sombre, et pour seul accident ou événement une cavité apparaît au loin, créée par un effet de lumière, venant à peine troubler I'horizontalité et la densité. Le thème de la naissance, la terre comme origine et matrice, est ici inséparable du thème de la mortalité; la froideur de la terre, sa densité et son odeur évoquent tout autant une tombe. Cette réflexion sur l'appartenance et la séparation qu'évoquent ici la naissance et la mort, Lani Maestro la concilie à sa condition d'exilée:

Avec l'expérience du déplacement, disait-elle à propos de Souffle blessé, existe un désir mélancolique, celui d'un souvenir qui prend forme. L'objet de ce désir prend ses racines dans ce semblant de chez-soi qui constitue le ventre de la mère. Les quêtes d'identité constituent toutes de profonds désirs de chez-soi. Nous sommes tous des exilés du fait que nous sommes venus au monde. ${ }^{6}$

Cette expérience de l'exil motive également l'installation Cradle (Couche, 1996), qui évoque encore plus avec force l'idée de la demeure, le chez-soi, comme origine et fondement de l'appartenance, mais aussi comme séparation. Ce parallèle entre l'expérience de l'exil et la mélancolie, Julia Kristeva l'a déjà tracé lorsqu'elle disait dans Soleil noir que «le mélancolique est un étranger dans sa langue maternelle» ${ }^{7}$. De manière à remplir complètement une pièce, de petites habitations de toile de moustiquaire et de nattes de palme sont suspendues dans l'espace par des fils tendus aux murs et au plafond. Cette œuvre arrive à évoquer à la fois l'isolement et l'idée de communauté, et la demeure devient le lieu d'une mémoire et d'une commémoration, une possible survivance. Trinh T. Minh-ha a réfléchi sur la notion de demeure du point de vue de l'exilé et du migrant, en la définissant comme le lieu d'une «mémoire à venir». Sa réflexion est très proche de ce que Lani Maestro tente ici d'évoquer:

[...] le langage ne peut vivre et se renouveler qu'en s'hybridant et qu'en transformant ses propres règles comme s'il migrait dans le temps et l'espace. La demeure pour l'exilé et le migrant ne peut guère être plus qu'un lieu transitoire et circonstanciel, puisque son «origine» ne peut être retrouvée, et puisque sa présence/absence ne peut être entièrement bannie de la demeure « reconstruite». M étaphoriquement et littéralement parlant, ce sont les aller-retour entre le chez-soi et l'extérieur qui deviennent la manière d'habiter. Chaque mouvement entre ici et là supporte patiemment un mouvement à l'intérieur de l'ici et un 
mouvement à l'intérieur de là. En d'autres mots, le retour est aussi un trajet dans la couche d'une «mémoire à venir». 8

La présence et l'absence se traduisent souvent chez Lani Maestro par le silence et le vide. Signes d'un possible effacement de la mémoire, ils posent paradoxalement la nécessité de cette même mémoire. Plusieurs œuvres, en effet, font entendre ce silence nous incitant à faire l'expérience de l'absence. I want! I want! I want! est un bel exemple. La bande sonore alterne entre un temps de silence et un temps sonore où se mêlent des voix et des rires que l'on peut facilement confondre avec des pleurs; ces deux intervalles s'inversent selon le moment de notre entrée dans l'espace d'exposition. Calme, tranquilité, puis soudainement la confusion des voix vient remplir cet espace vide. La bande sonore de l'installation Pulse (1998) fonctionne de façon similaire: offrant de prime abord un lieu calme, paisible, I'habitat est perturbé et envahi par une agitation de bruits d'oiseaux.

Par des ouvertures relativement petites dispersées çà et là dans la structure architecturale qui compose I want! I want! I want!, on peut voir des échelles qui vont dans toutes les directions, brouillant les perspectives et les points de vue malgré l'extrême symétrie du cube blanc. Ces échelles n'évoquent pas tant le désordre que le lointain et l'impossibilité de résorber la distance malgré le désir. L'installation fait référence à une gravure de William Blake du $18^{\mathrm{e}}$ siècle représentant un homme sous une échelle qui, la nuit, lève les bras vers le ciel. Au bas de la gravure apparaît cette légende écrite à la main: «I want! I want!».

Toujours confronté à l'autre et à cet inaccomplissement du désir, toujours pris à gérer les proximités autant que les distances - le proche comme le lointain pour reprendre les mots de Benjamin -, le spectateur y perd ses propres repères, ses propres règles. L'intermédialité ne sert pas qu'à brouiller les spécificités qui définissent les médiums et les identités avec le modernisme, elle déstabilise le rapport du spectateur à ce qu'il voit, à ce qu'il est, son rapport à l'autre et au monde. Les installations de Lani M aestro rattachent cette «notion » à l'idée d'interstice et de migration, elles nous forcent à penser les croisements en termes d'espace entre, et surtout en termes de mouvement et de déplacement, c'est-à-dire d'espace et de temps.

1. S. Horne, Lani Maestro. Le Souffle blessé, Q uébec, La chambre blanche, 1996, p. 31.

2. Je renvoie notamment aux textes sur Baudelaire et aux Thèses sur le concept d'histoire, où la figure de l'Ange à la fois pose les limites de l'H istoire et représente une modernité «déchirée» (C. Perret). Également, en développant son concept d'allégorie en opposition au symbole, Walter Benjamin se débarrasse de l'expressionnisme, il est critique d'une littérature dont l'enjeu est le narcissisme mélancolique du sujet; le renoncement qu'implique le deuil ne se ramène pas à une quelconque forme de repli, au contraire, il s'affiche comme détachement et résistance. "C'est la culpabilité [disait-il dans L'O rigine du drame baroque allemand] qui interdit au signifiant allégorique de trouver en lui-même l'accomplissement de sa signification. A l'aveu du manque, au deuil de ce qui manque, I'allégorie préfère la culpabilité d'un crime imaginaire; c'est là sa mélancolie». Voir C. Perret, Walter Benjamin sans destin, Paris, La Différence, 1992, p. 39.

3. À propos de cette dimension dialectique et politique de la mélancolie, voir l'ouvrage de M. Pensky, Melancholy Dialectics. Walter Benjamin and the Play of M ourning, Amherst, The U niversity of Massachussets Press, 1993, p. 11-13.

4. G. Koh, «Lani Maestro», dans Traversées, O ttawa, Musée des beaux-arts du Canada, 1998, p. 143 et 146.

5. S. Horne, op. cit, p. 40.

6. Ibid, p. 36.

7. J. Kristeva, Soleil noir. Dépression et mélancolie, Paris, Gallimard, 1987.

8. Trinh T. Minh-ha, «O ther than myself/my other self», dans Travellers' Tales: Narratives of Home and Displacement, Londres et $\mathrm{N}$ ew York, Routledge, 1994, p. 14-15 (c'est moi qui traduis). 


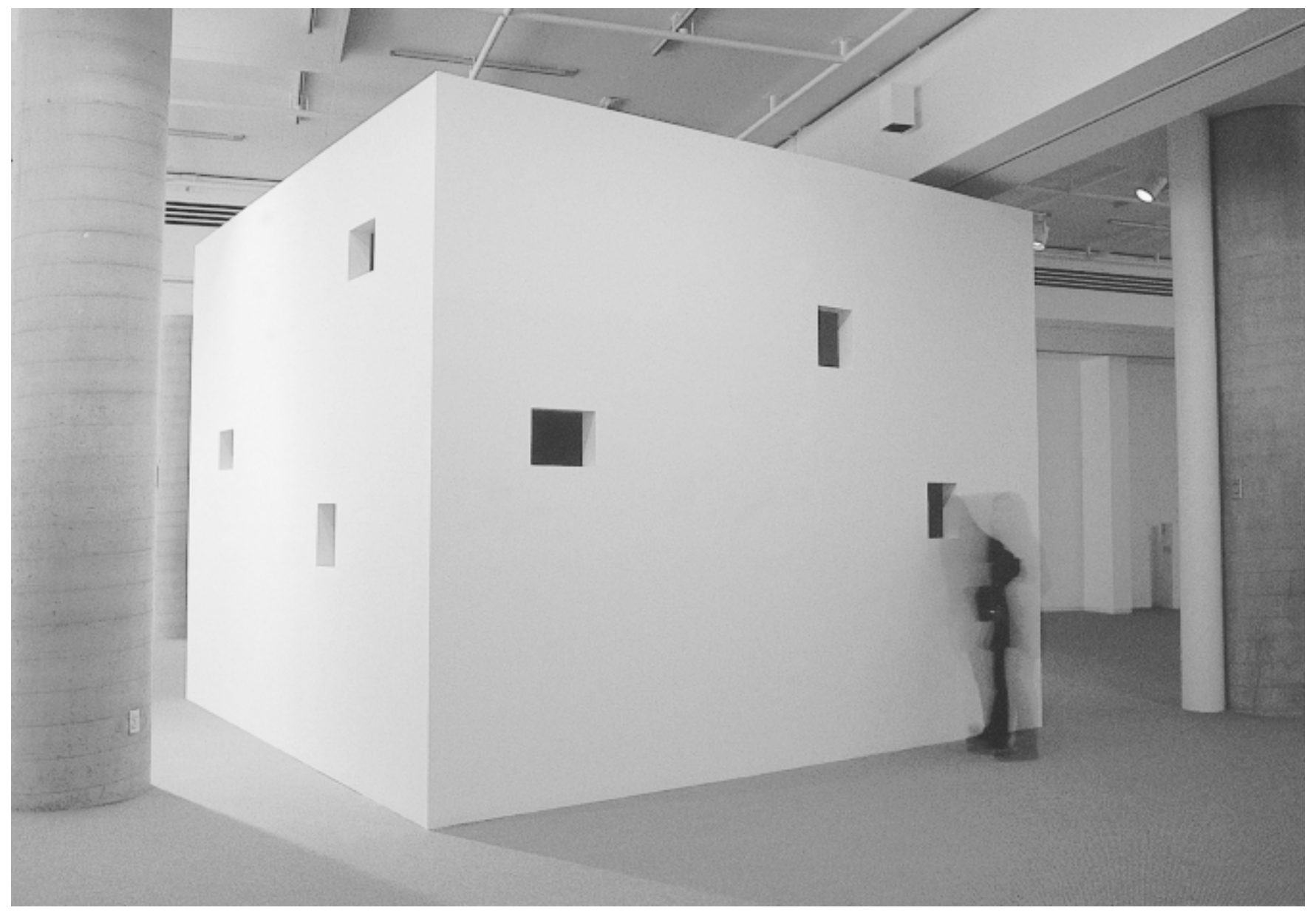




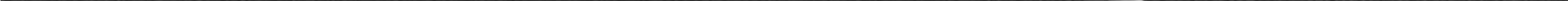




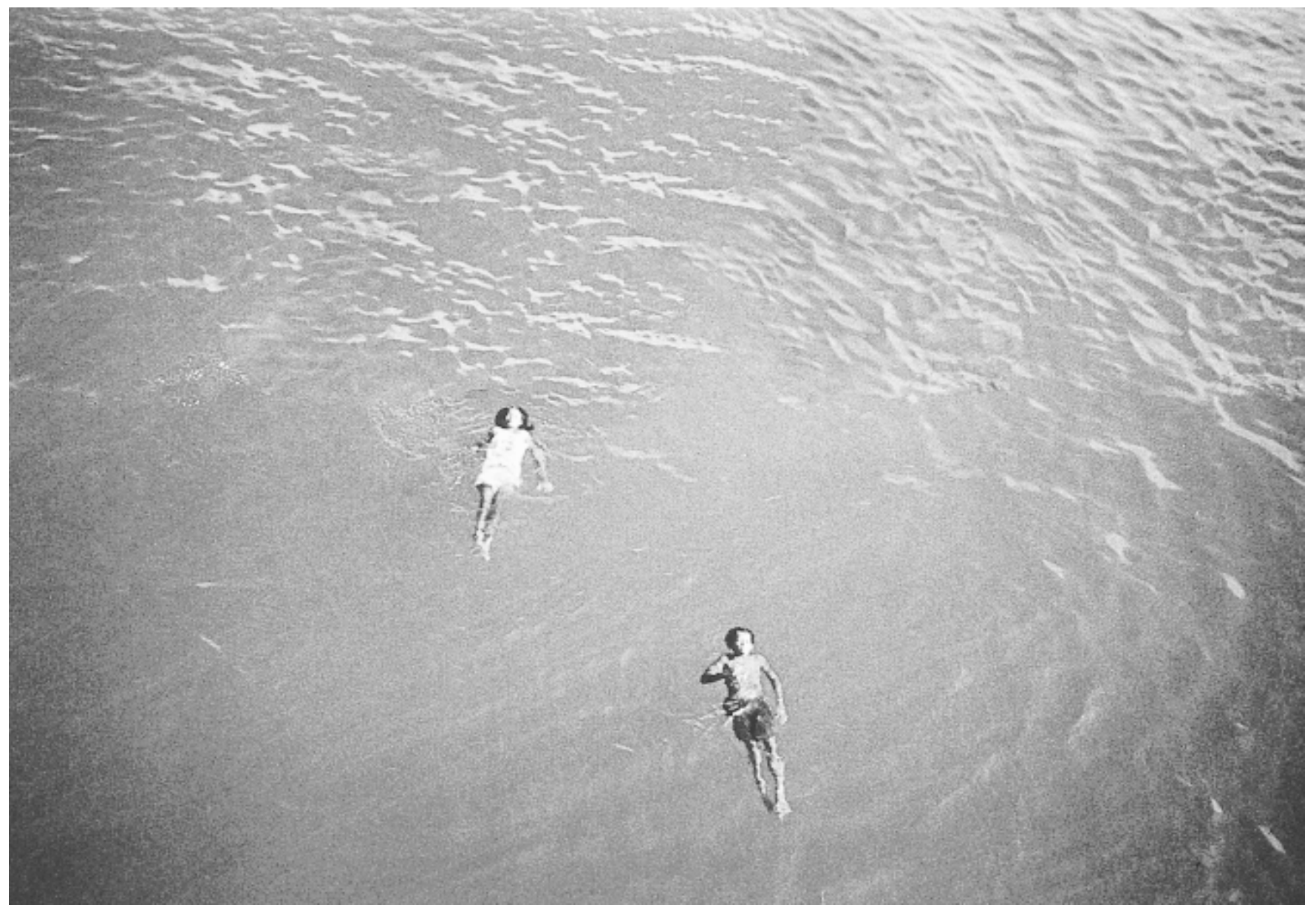




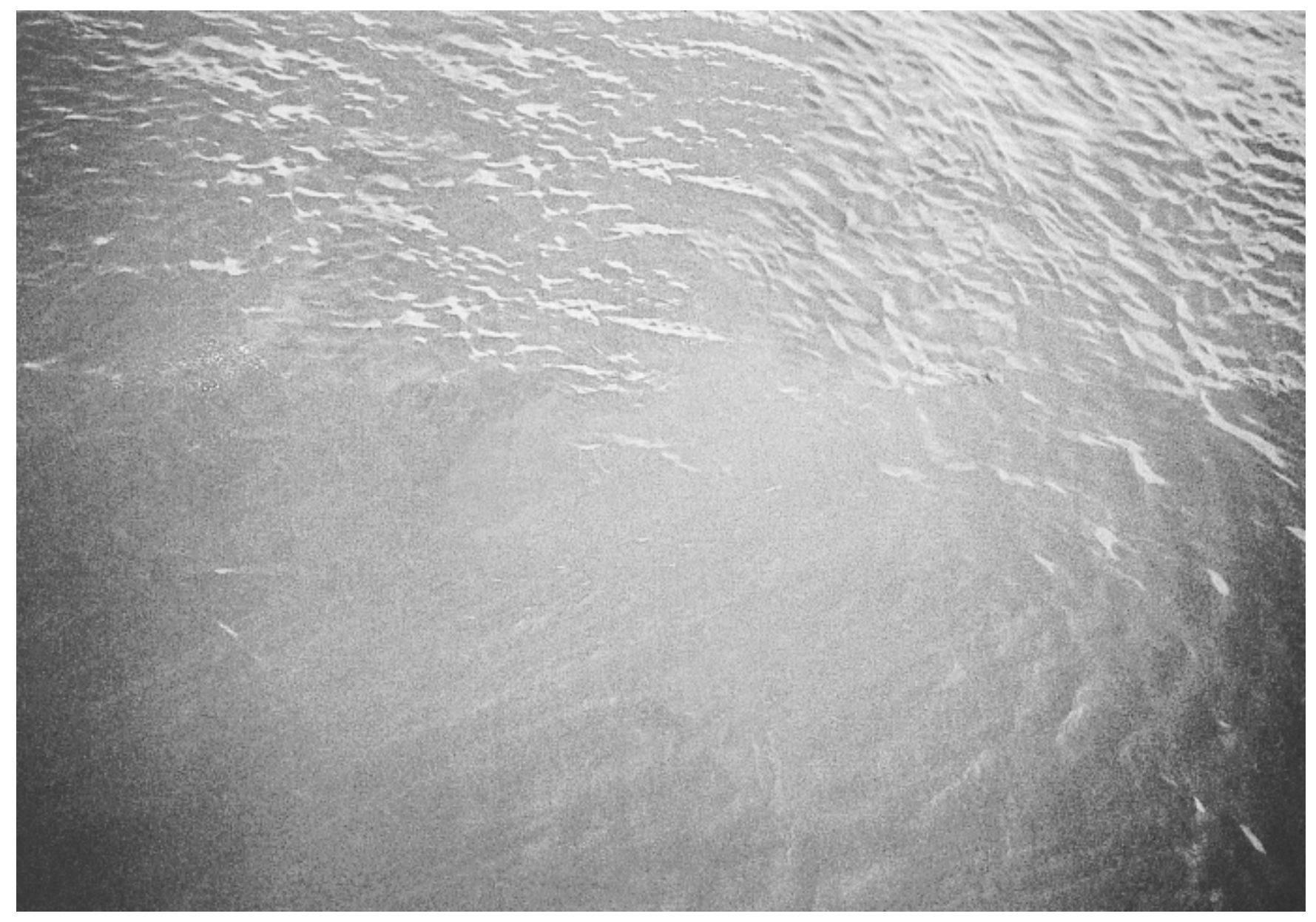




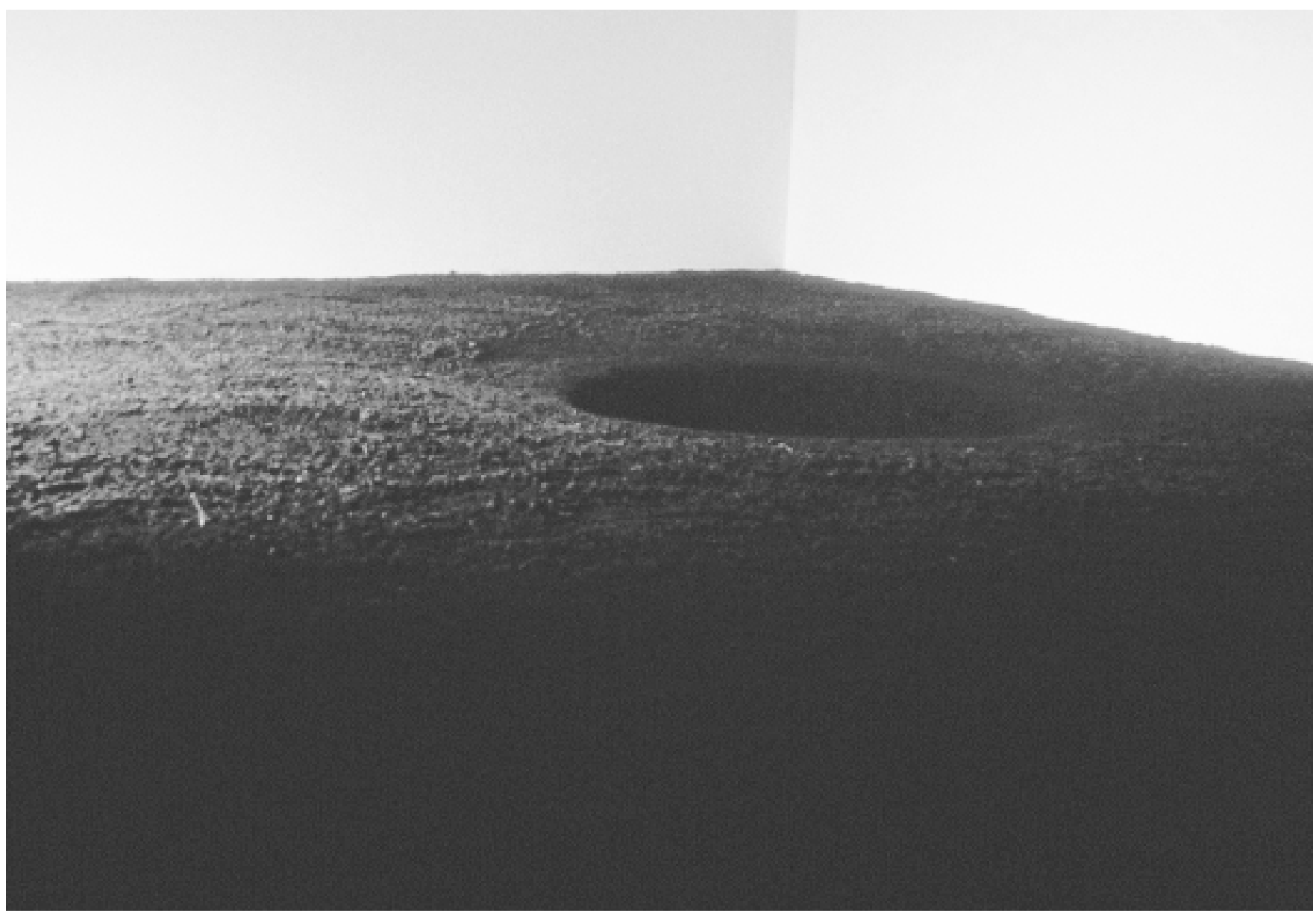




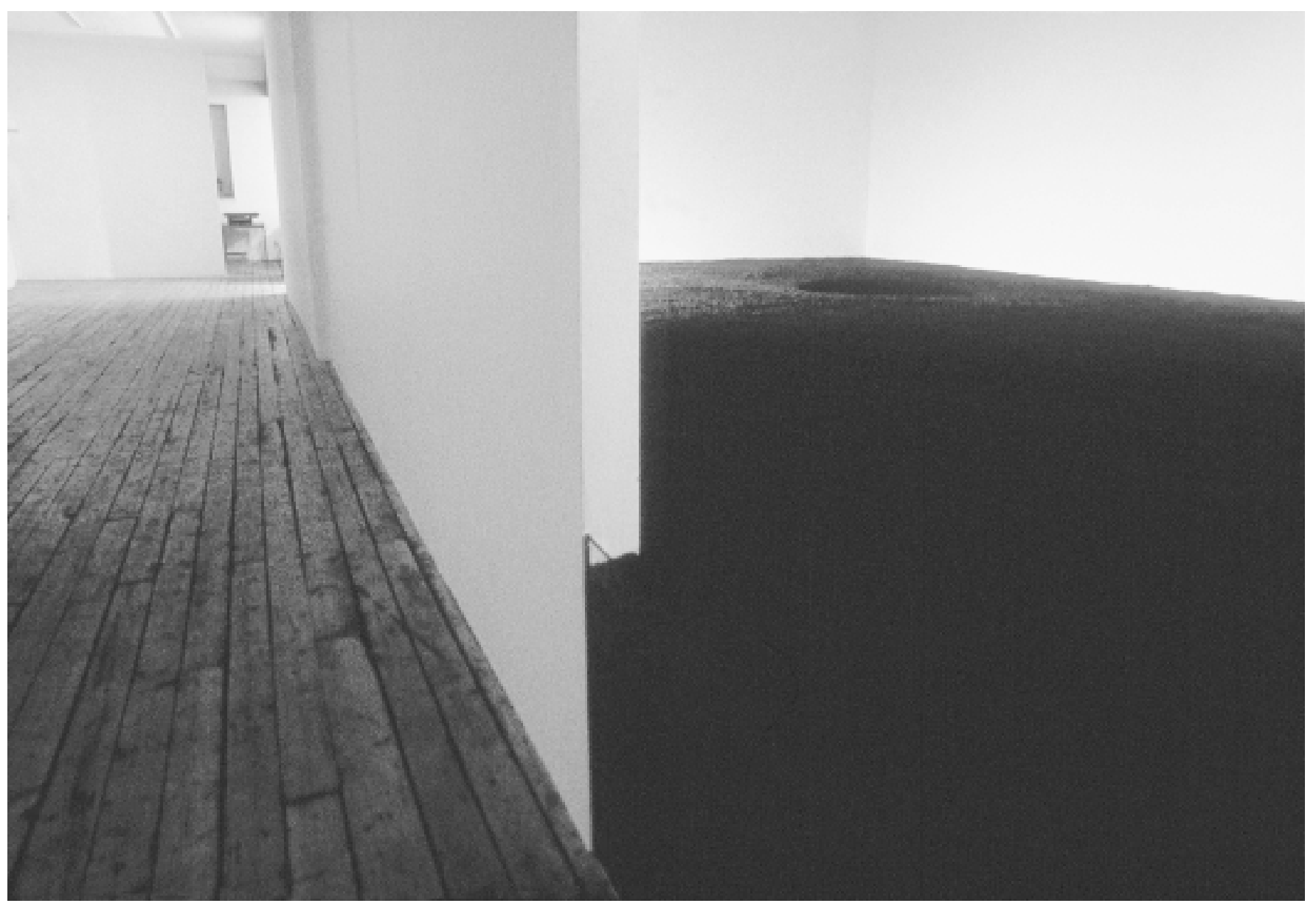




\section{Les installations}

Cradle (Couche), 1996.

Étamine, ficelles de sisal, nattes de palme.

Dimension variable.

Présentée lors de l'exposition Crossings/Traversées, Musée des beaux-arts du Canada, 0 ttawa.

Photo: Elizabeth Davis.

I want! I want! I want!, 2000.

Échelles de pin taillées à la main, structure de gypse et métal, moniteurs audio, lumière.

Structure: $13^{\prime} \times 13^{\prime} \times 14^{\prime}$; échelles: 14'.

Galerie de I'U Q AM, M ontréal.

Photo: Paul Litherland.

Dream of the other (Rêve de l'autre), 1998.

Diapositives, 2 projecteurs de diapositives, bande sonore.

Galerie La Centrale, Montréal, Q uébec.

Photo: Lani Maestro.

Le Souffle blessé (A Wound in the lung), 1994.

Terre, bois, verre et gypse.

La Chambre blanche, Q uébec.

Photo : Ivan Binet.

Taema, 2000.

Détail de l'installation.

Livre, bois, verre, vidéo.

Gendai Gallery O ntario.

Photo: Paul Litherland.
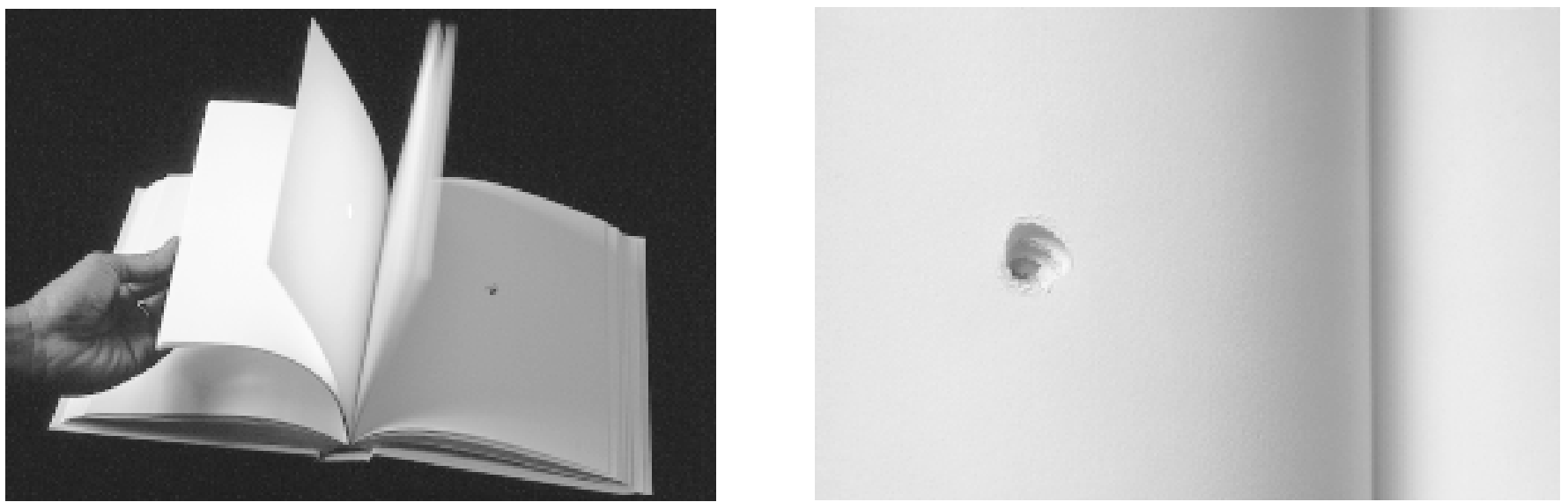